CARTA AL EDITOR

\title{
Breves comentarios acerca de las Guías NeuroALAD para el diagnóstico y tratamiento de la polineuropatía diabética sensoriomotora recientemente publicadas
}

José A. Mesa-Pérez ${ }^{1 *}$, Ariel Odriozola², Ricardo Antonucci ${ }^{3}$ y Dan Ziegler $^{4}$

'Universidad Autonoma "Benito Juarez". Presidente Asociación Latinoamericana de Diabetes, Coordinador del Grupo NeuroALAD, Oaxaca de Juárez, México; ${ }^{2}$ Editor de Guías NeuroALAD, Instituto Catalán de Endocrinología y Nutrición, Barcelona, España; ${ }^{3}$ Vicecoordinador del Grupo NeuroALAD, Dr. Ricardo Antonucci, División Nutrición, Hospital de Clínicas José de San Martín, Buenos Aires, Argentina; ${ }^{7}$ German Diabetes Center at the Heinrich Heine University, and the Department of Metabolic Diseases, University Hospital, Dusseldorf, Alemania

Estimado editor de la revista ALAD:

Después de revisar las guías para el diagnóstico y tratamiento de la polineuropatía diabética sensoriomotora (DSPN) recientemente publicadas en la revista $A L A D$ es nuestro interés como grupo NeuroALAD esclarecer dos temas ajenos a la editorial que usted coordina:

1) En la guía se hace referencia al «tratamiento del dolor nociceptivo", término que no consideramos adecuado para el asunto que se trata, y que es interés del grupo sea sustituido por el de «tratamiento del dolor neuropático», ya que es el tipo de dolor asociado con la DSPN. La corrección se fundamenta en que el término «dolor nociceptivo» se refiere al dolor transitorio en respuesta a un estímulo nocivo, mientras que el «dolor neuropático» se refiere al dolor espontáneo y a la hipersensibilidad asociada a este con daño o lesión del sistema nervioso ${ }^{2}$. Debido a que las recomendaciones de tratamiento descritas en las guías ALAD se aplican al dolor neuropático en pacientes con DSPN y no al dolor nociceptivo, consideramos necesario hacer la aclaración.

2) En la página 15 la guía recomienda el uso de benfotiamina preferentemente en pacientes tratados de forma prolongada con metformina (absorción reducida de cianocobalamina). Este concepto debe corregirse por varias razones: a) la cianocobalamina es una forma sintética de la vitamina B12, y un ingrediente de suplemento; b) existe evidencia que indica que el tratamiento con metformina se asocia con niveles más bajos de vitamina B12, lo que conduce a una deficiencia franca o un estado límite en algunos pacientes con diabetes tipo $2^{3}$; la benfotiamina es un derivado sintético del S-acilo soluble en lípidos (profármaco) de tiamina (vitamina B1); de forma particular, la concentración plasmática baja de tiamina parece ser prevalente en pacientes con diabetes tipo 1 y tipo 2 asociada con un aumento del aclaramiento de tiamina ${ }^{4}$, pero no del 
tratamiento con metformina, y c) no obstante lo comentado, existe un contexto en el que la interacción de metformina y tiamina parecen desempeñar un papel. Existen datos experimentales que sugieren que el transportador de cationes orgánicos 1 (OCT1), que se expresa abundantemente en el hígado humano, desempeña un papel importante en la disposición del fármaco y representa el principal transportador de absorción hepática para metformina 5 . El transportador de tiamina 2 (THTR-2), que se expresa altamente en el intestino delgado, regula tanto la absorción de tiamina intestinal como de metformina ${ }^{6}$. Dado que la metformina inhibe la absorción de tiamina mediada por OCT1 y THTR-2 $2^{5,6}$ y aunque en teoría tal interacción es posible, faltan datos en huma$\operatorname{nos}^{6}$. No existe limitación en el uso de benfotiamina en pacientes diagnosticados con DSPN y aquellos con deficiencia de vitamina B1 pueden beneficiarse en particular.

\section{BIBLIOGRAFÍA}

1. Mesa JA, Odriozola A, Antonucci R, Campillo N, Márquez G, Gil JC, et al. Guías ALAD basadas en evidencias para el diagnóstico y el tratamiento de la polineuropatía sensitivomotora diabética 2019 (Grupo NeuroALAD). Rev ALAD. 2019;9:1-27.

2. Woolf CJ. Dolor: pasar del control de los síntomas al manejo farmacológico específico del mecanismo. Ann Intern Med. 2004;140:441-51.

3. Chapman LE, Darling AL, Brown JE. Asociación entre metformina y deficiencia de vitamina B12 en pacientes con diabetes tipo 2: una revisión sistemática y un meta-análisis. Diabetes Metab. 2016;42:316-27.

4. Thornalley PJ, Babaei-Jadidi R, Al AH, Rabbani N, Antonysunil A, Larkin J, et al. Alta prevalencia de baja concentración plasmática de tiamina en la diabetes relacionada con un marcador de enfermedad vascular. Diabetologia. 2007;50:2164-70.

5. Chen L, Shu Y, Liang X, Chen EC, Yee SW, Zur AA, et al. OCT1 es un transportador de tiamina de alta capacidad que regula la esteatosis hepática y es un objetivo de la metformina. Proc Natl Acad Sci USA. 2014;111:9983-8.

6. Liang X, Chien HC, Yee SW, Giacomini MM, Chen EC, Piao M, et al. La metformina es un sustrato e inhibidor del transportador de tiamina humano, THTR-2 (SLC19A3). Mol Pharm. 2015;12:4301-10. 